\title{
LV Baden-Württemberg: Befragung zur Pflegekammer geplant
}

Am 21. Juli 2017 fand in Stuttgart die Mitgliederversammlung des Landesverbands Baden-Württemberg statt. Mit über 60 Teilnehmenden war die Mitgliederversammlung sehr gut besucht. Den Auftakt gestaltete die Staatssekretärin im Ministerium für Soziales und Integration, Bärbl Mielich. Sie würdigte die immense Bedeutung der Pflege und der Pflegeausbildungen und stellte die Positionen des Sozialministeriums zur Novellierung des Pflegeberufegesetzes und zur Pflegekammer dar. In der anschließenden, lebhaften Diskussion betonten die Mitglieder, dass für die Umsetzung eines Rahmenlehrplans auf Landesebene und für die curriculare Arbeit der Schulen ein ausreichendes Zeitfenster vorgesehen werden muss. Deutlich wurde der Wunsch nach einem verbindlichen Landeslehrplan formuliert und die Beibehaltung des derzeit gültigen Lehrer-Schüler-Verhältnisses von 1:15 bis 1:18 gefordert.

Im Anschluss stellte Julia Köppen vom Fachgebiet Management im Gesundheitswesen der TU Berlin die Ergebnisse der Followup-Studie RN4Cast (Registered Nurse Forcasting) zur Arbeitssituation der Pflegenden in deutschen Krankenhäusern im internationalen Vergleich vor. Schon 2010 war deutlich geworden, dass Deutschland im Vergleich zu anderen an der Studie beteiligten Ländern eine schlechtere Pflegepersonalausstattung aufzubieten hat; dies hat Auswirkungen auf die Qualität der Versorgung und die Arbeitszufriedenheit der Pflegenden. Unter anderem konnte nachgewiesen werden, dass über 30\% der Pflegenden unter emotionaler Erschöpfung (Burnout) leiden. Die deutsche Nachfolgestudie (2015) zeigt, dass sich nahezu alle Ergebnisse weiter verschlechtert haben.

Im Bericht des Landesvorstands wurden die vielfältigen Aktivitäten, insbesondere im Landespflegerat zur Pflegekammer, zur Wiederaufnahme der Arbeit der AG Ethik und zur Eingruppierung der Pflegepädagogen vorgestellt. Für die nächsten Monate steht die weitere Arbeit an der Homepage, Angebote für Praxisanleiter und die Bildung einer Arbeitsgruppe "Landeslehrplan" auf der Agenda.

Am Nachmittag stellten Christa Rustler und Barbara Driescher das Programm „Astra-Plus - Aktive Stressprävention und Rauch-

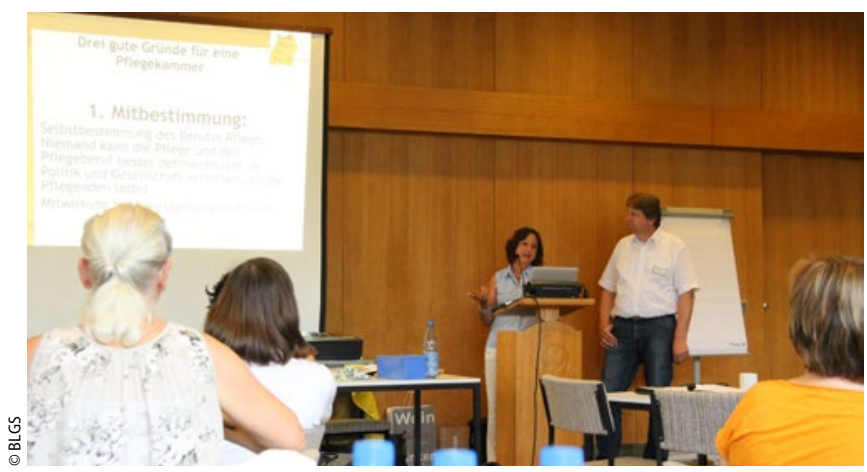

freiheit in der Pflege" vor. Der Astra-Plus Kurs hat die Gesundheitskompetenz von Auszubildenden in den Pflegeberufen, insbesondere zum Rauchen, im Fokus. Aus der Arbeitsgruppe "Prüfungen" berichtete Christine Maier über den Stand der Diskussion zu den fallbasierten Aufgaben. Zukünftig ist vorgesehen, die Operatoren in den Fragestellungen einheitlich zu definieren.

Die Tagung endete mit einer Multiplikatorenschulung für Kollegen, die die Gestaltung von Veranstaltungen zur Pflegekammer übernehmen werden. Barbara Driescher und Hans Schollenberger stellten eine gemeinsam mit dem Landespflegrat entwickelte Präsentation vor, die für die Gestaltung von Veranstaltungen dieser Art an Schulen, Krankenhäusern und Pflegeeinrichtungen genutzt werden kann. Ergänzend dazu steht nun den Mitgliedern des Landesverbandes ein von Martin Boochs entwickelter Unterrichtsentwurf zu diesem Thema zur Verfügung.

Da bis Ende des Jahres eine Befragung der Pflegenden in Baden Württemberg zur Errichtung einer Landespflegekammer geplant ist, ist es dem Landespflegerat ein großes Anliegen, an der umfassenden Information der Pflegenden im Land mitzuwirken.

Kirsten Heiland

Landesvorstand Baden-Württemberg

\section{Niedersachsen/Bremen: Mehr Qualität - mehr Qualifikation}

In Hannover fand am 14. Juni 2017 die Tagung und Mitgliederversammlung des Landesverbandes Niedersachsen/Bremen statt. Im Schatten des beschlossenen Pflegeberufegesetzes betonte Christine Vogler, stellvertretende Vorsitzende des BLGS e.V., zu Anfang, wie wichtig es sein wird, in der Pflegeausbildung, unter Berücksichtigung des pflegewissenschaftlichen Fortschrittes, Kompetenzen zur Pflege von Menschen aller Altersgruppen in allen Pflegesettings zu vermitteln und damit übergreifende pflegerische Qualifikationen zu erreichen. Es gilt, sich den kommenden Herausforderungen gemeinsam zu stellen.

Jacques Doillet, Artset Forschung Bildung Beratung GmbH, stellte das Qualitätsmodell LQW vor. Diese Methode der Lernorientierten Qualitätsentwicklung in der Weiterbildung/Bildung wurde mit Mitteln des Bundeministeriums für Bildung und Forschung und des europäischen Sozialfonds gefördert. Dem Modell der Lerner- und kundenorientierten Qualitätstestierung liegt der Gedanke zugrunde, dass Qualitätsmanagement nicht vorrangig ein technisches Verfahren, sondern als umfassende Organisationsentwicklung und Qualität zu verstehen ist.
Auf der nachmittäglichen Mitgliederversammlung stand die Neuwahl des Vorstandes im Mittelpunkt. Michaela Picker eröffnete die MV mit dem Jahresbericht des Landesverbandes. Anschließend fand die Neuwahl des Vorstandes statt: Birgit Burkhardt, Schulleiterin am Pius-Hospital in Oldenburg, löst Michaela Picker, Schulleiterin an den Städtischen Klinikum Braunschweig, im Vorsitz ab.

Frau Picker begleitete dieses Amt seit dem 22.11.2012 und trat aus privaten Gründen nicht wieder zur Wahl an. Der neue Vorstand bedankt sich für ihr Engagement im Landesverband und für die vertrauensvolle und unterstützende Zusammenarbeit!

Michael Krol (Geschäftsbereich Bildungsakademie - Gesundheit nordgGmbH) wurde als stellv. Vorsitzender gewählt; weitere Mitglieder sind: Kerstin Bugow (Schulleiterin - Pflegeschule der Med. Hochschule Hannover), Anja Koch (Schulleiterin - Pflegeschulzentrum Goslar) und Barbara Venhaus-Schreiber (Schulleiterin - Bremer Krankenpflegeschule der freigemeinnützigen Krankenhäuser e.V.).

Birgit Burkhardt Landesvorstand Niedersachsen/Bremen 